\title{
The Abundant Irregular Satellites of the Giant Planets
}

\author{
Scott S. Sheppard and David C. Jewitt \\ Univ. of Hawaii, Institute for Astronomy, 2680 Woodlawn Dr., \\ Honolulu, Hi 96822, USA
}

\begin{abstract}
Irregular satellites have eccentric orbits that can be highly inclined or even retrograde relative to the equatorial planes of their planets. These objects cannot have formed by circumplanetary accretion as did the regular satellites which follow un-inclined, nearly circular, pro-grade orbits. Instead, they are likely products of early capture from heliocentric orbit. The study of the irregular satellites provides a unique window on processes operating in the young solar system. Recent discoveries around Jupiter (45 new satellites), Saturn (13), Uranus (9), and Neptune (5) have almost increased the number of known irregular satellites by a factor of ten and suggest that the gas and ice giant planets all have fairly similar irregular satellite systems. Dynamical groupings were most likely produced by collisional shattering of precursor objects after capture by their planets. Jupiter is considered as a case of special interest. Its proximity allows us to probe the fainter, smaller irregular satellites to obtain large population statistics in order to address the questions of planet formation and capture.
\end{abstract}

\section{Discussion}

The recent development of sensitive, large scale $\mathrm{CCD}$ detectors has refreshed the study of irregular satellites by enabling a new wave of discovery. Large fields of view are needed because the Hill spheres (the regions of gravitational influence about each planet in which satellites could be stable) are large (see Table 1). Sensitivity is needed because the majority of irregular satellites are small and therefore faint. Also for this reason, the irregular satellite system of Jupiter has been a particular target of recent study and its currently the best characterized (Sheppard and Jewitt 2003). Each planet possesses both retrograde and pro-grade outer satellites, with the former being the more numerous. Irregular satellites in retrograde orbits have semi-major axes that are all less than $0.48 r_{H}\left(0.33 r_{H}\right.$ for the pro grades), where $r_{H}$ is the Hill sphere radius. Some satellites currently have apojoves up to $0.65 r_{H}$. Satellites with inclinations $55<i<130$ are absent, probably a result of the Kozai effect that couples variations in inclination and eccentricity and forces highly inclined satellites to reach large eccentricities and makes them unstable (Carruba et al. 2002). The preponderance of retrograde satellites at larger distance is probably a result of resonant perturbations by the Sun (Nesvorny et al. 2003). At present it is practically impossible for planets to permanently capture satellites since no efficient dissipation mechanism exists. Satellite capture could have occurred more easily towards the end of a planet's formation due to gas drag from an extended atmosphere, the enlargement of the Hill sphere caused by the planet's mass growth and/or higher collision probabilities with nearby small bodies (Colombo \& Franklin 1971; Heppenheimer \& Porco 1977; Pollack et al. 1979). If so, 
satellite capture occurred on the same timescale as the planet's growth. While Jupiter currently possesses the largest number of known satellites of any of the planets (Sheppard \& Jewitt 2003), the other giant planets also have significant irregular satellite populations (Gladman et al. 2000 and 2001; Holman et al. 2003; Sheppard et al. 2003). All are thought to have been captured by their respective planets. In common with Jupiter, the other systems show dynamical grouping, but the satellites of more distant planets are fainter and observationally less well characterized. In particular, Jupiter shows groups in semi-major axis and inclination space while other planets show grouping mostly in inclination space. It is yet to be determined if the groupings in inclination space are because of resonant effects or not. In many of the groups a few large satellites are accompanied by many smaller ones, the latter having a steep size distribution similar to that of the main-belt asteroid families. By extrapolation, we estimate that, within a factor of two, each planet possesses approximately 100 irregular satellites with diameters greater than $1 \mathrm{~km}$. We find that the four giant planets possess about the same number of irregular satellites and groupings with no dependence on the planet's mass when comparing them to the same limiting size of satellites. This is especially remarkable given that the ice giants Uranus and Neptune may have had formation histories quite different from the gas giants Jupiter and Saturn. It is unlikely that the satellite groups were produced by the sole action of aerodynamic forces during capture, because self-gravity would prevent the fragments from dispersing (Pollack et al. 1979). Additionally, gas drag acting on the fragments would produce size-dependent sorting of the orbits within each group that is not observed. For these reasons we believe that the disruptions occurred after capture and after the dissipation of the gas left over from planet's formation. Fragmentation of the parent satellites could be caused by impact with interplanetary projectiles (principally comets) or by collision with other satellites, assuming both were once much more numerous than now. For an in depth review of irregular satellites see Jewitt et al. (2003).

Table 1. Irregular Satellites of the Giant Planets

\begin{tabular}{cccccc}
\hline Planet & $\begin{array}{c}\text { irreg. } \\
\text { sats. } \\
(\#)\end{array}$ & groups & $\begin{array}{c}\text { limiting } \\
\text { radii } \\
(\mathrm{km})\end{array}$ & $\begin{array}{c}\text { Hill } \\
\text { Radii } \\
(\mathrm{deg})\end{array}$ & $\begin{array}{c}\text { Hill } \\
\text { Area } \\
\left(\mathrm{deg}^{2}\right)\end{array}$ \\
\hline Jupiter & 53 & 6 & 3 & 4.7 & 70 \\
Saturn & 14 & $4-5$ & 8 & 3.0 & 28 \\
Uranus & 9 & $2-3$ & 16 & 1.5 & 7 \\
Neptune & 7 & 4 & 34 & 1.5 & 7
\end{tabular}

\section{References}

Carruba, V. et al. 2002, Icarus, 158, 434

Colombo, G. \& Franklin, F. 1971, Icarus, 15, 186

Gladman, B. et al. 2000, Icarus, 147, 320

Gladman, B. et al. 2001, Nature, 412, 163

Heppenheimer, T. \& Porco, C. 1977, Icarus, 30, 385

Holman, M. et al. 2003, IAU Circ., 8047 
Jewitt, D., Sheppard, S., \& Porco, C. in press, in Jupiter II, ed. F. Bagenal, Cambridge University Press, Cambridge, p. 236

Nesvorny et al. 2003, AJ, 126, 398

Pollack, J., Burns, J. \& Tauber, M. 1979, Icarus, 37, 587

Sheppard, S. \& Jewitt, D. 2003, Nature, 423, 261

Sheppard, S. et al. 2003, IAU Circ., 8193 